\title{
Numerical Study of Bacterial Influences Caused by Patient Breathing
}

\author{
Alireza Khademi ${ }^{1}$, Mohammad Hassan Saidi ${ }^{2}$, Masoud Darbandi ${ }^{1,3}$, Gerry E. Schneider ${ }^{4}$ \\ ${ }^{1}$ Department of Aerospace Engineering, Centre of Excellence in Aerospace Systems, Sharif University of Technology \\ Tehran, Iran \\ khademi_alireza@ae.sharif.edu; darbandi@sharif.edu \\ ${ }^{2}$ Sharif Energy Research Institute (SERI), Sharif University of Technology \\ Tehran, Iran \\ saman@sharif.edu \\ ${ }^{3}$ Institute for Nanoscience and Nanotechnology, Sharif University of Technology \\ Tehran, Iran \\ darbandi@sharif.edu \\ ${ }^{4}$ Department of Mechanical and Mechatronics Engineering, University of Waterloo \\ Waterloo, Ontario, N2L 3G1, Canada \\ gerry.schneider@uwaterloo.ca
}

\begin{abstract}
This research aims two major objectives: first, the influence of respiration on the particles concentration is investigated numerically in a cleanroom with a specified geometry and second, the respiration and the manner of particles diffusion are simulated. Generally, in many hospital cleanrooms such as the open-heart surgery, organ transplantation and neurosurgery rooms, reduction of the pollutant particles is important as a factor that can lead to capillary clogging during operation. In addition, the significance of reducing the concentration of large particles reveals more according to the fact that dust particles may act as a means for various pathogenic bacteria and viruses. Every bacterium has a dimension between 0.5 and 5 micrometers and the dimension of each virus is between 20 and 300 nanometers. Thus, it is evident that at least 1 bacterium and 15 viruses can fit on a 5-micrometer particle.
\end{abstract}

Keywords: Cleanroom, Bacterial Influences, Patient Breathing, Particle Tracking.

\section{Introduction}

A cleanroom is usually an environment where some activities are performed in order to produce products or carry out scientific and industrial researches and the amount of environmental pollutants is much lower than the common level of a closed environment. According to the definition given in ISO standard 14644-1, a cleanroom is a space where the concentration of the particles is controlled and it is constructed and operated such that the admittance, generation and settlement of the particles in the environment are minimized. Since the distribution of the particles concentration should be determined in order to understand properly the condition of the flow circulation, thus many numerical researches focus on the effective parameters in the manner of the particles distribution in a cleanroom that are in fact considered as the effective parameters in the design and fabrication. Some of the most prominent researches are presented in the following.

Shimada et al. [1] and Saidi et al. [2] have observed the influence of location of the particle generation source and the effect of the inlet and outlet gates arrangement on the amount of the particles concentration in their researches. The obtained results imply the remarkable effect of pollutant source location and the selection procedure for the arrangement of the inlet and outlet gates on the amount of the pollutants concentration. In research performed by Saidi et al. [2], the impact of the pollutant particles generation source displacement on the distribution of the suspended particles is realized. The achieved outcomes indicate that the specifications of the concentration distribution such as the final efficiency and the propagation radius have increased. When the movement path of the pollutant source coincides with the movement path of the air conditioner streamlines, the final efficiency represents the minimum amount of dependency on the movement path of the pollutant source. In a research work accomplished by Méndez et al. [3] and Zhong et al. [4], the effect of the inlet and outlet gates location on the airflow pattern in clean spaces are found. It is seen that the location of the inlet gates has a significant influence on the distribution of the flow specifications such as the velocity, temperature and concentration of 
the pollutants while the position of the outlet gate has a negligible effect. According to research works carried out by Hagström et al. [5] and Lee and Awbi [6], the location of the obstacles and equipment drastically affects the flow pattern in the ambient. However, the results of these researches are very general with respect to the diversity of the cleanrooms applications.

In many of the performed subsequent researches, the effect of different parameters including the geometry of the inlet gates and the effect of particle generation from the floor (the effect of the particles suspension) is observed which are beyond the scope of this research. Airflow in cleanrooms usually is accomplished by some phenomena such as being a low velocity, turbulent, unsteady and having buoyancy effect [7]. This even holds for cleanrooms that are called layered cleanrooms. Understanding some characteristics of the turbulent flow is important for modelling the internal turbulent flow. Irregularity, permeability, three-dimensionality, and dissipation are among the important characteristics of turbulence. These characteristics prevent from the thorough comprehension of turbulence. Anyhow, the engineering real world such as the design of air conditioning systems requires accurate prediction of the turbulent flow.

In this study, according to the above mentioned research works, numerical simulations of the patient's breathing in the cleanroom and the distribution of particle concentrations are performed. This activity has not been reported in the previous past research efforts.

\section{The Computational Method}

According to the computational limitations, the eddy viscosity method should be utilized against the large eddy simulation method, the direct simulation method and the Reynolds stress based method. In addition, according to the importance of investigating the particles settlement at specific locations such as the patient's body in the environmental isolated room, the Eulerian-Lagrangian method is employed for simulating the concentration. With respect to the size and density of the studied particle, the virtual mass, pressure, Saffman and Brownian forces are neglected in this method. According to the fact that the walls are one of the main sources of vorticity in a flow, the wall effect should also be taken into consideration in order to enhance the calculations in the vicinity of the wall during the simulation by k- $\varepsilon$ model as the presence of wall significantly affects the turbulence level in the vicinity of the wall. According to the accuracy and capability of the RNG k- $\varepsilon$ turbulence model, it is used for turbulence modelling. The solution convergences in every step is achieved when the solution residuals decrease to $10^{-3}$ and $10^{-4}$, respectively for continuity and flow equations. With respect to the existence of a filter box or plenum box at the air inlet of the cleanroom and the large dimensions of the inlet gate, the velocity of the air entering the room is usually uniform. Constant velocity boundary condition with a velocity of 0.9 meter per second is considered at the inlet. No-slip condition holds on the walls and the standard wall function was used for applying the effects of the wall on the flow turbulence. At the flow exit, the outlet boundary condition is considered at the outlet gates that implies zero gradients for the flow variables. This is a reasonable assumption according to the continuity of the channel after the return gate and the existence of the return plenum.

In Eulerian-Lagrangian method, the fluid field is solved using the Eulerian method and the behaviour of the solid particles is studied from the Lagrangian point view. In this method, processes such as the particles settlement and diffusion are investigated thoroughly. The concentration simulation is commonly fulfilled by the two Eulerian-Eulerian and Eulerian-Lagrangian approaches. The flow field is solved by the Eulerian method in both approaches [8].

\section{The Test Case}

The cleanroom of the Mechanical Engineering Department of Sharif University of Technology is constructed to have a rectangular cross-section and approximate dimensions of $2.9 * 3.7$ meters and a height of 2.5 meters. It has six inlet gates in the ceiling with dimensions of $60 * 60$ square centimetres and five outlet gates on the walls with dimensions of $30 * 45$ centimetres and the inlet arrangement can be altered by selecting the different inlet gates in the ceiling.

Respiration is considered as a reciprocating flow (inhale and exhale) and a stop. The duration of each action is equal to 2.5 seconds and the respiration rate is 0.25 meters per second. In this analysis, the particles leaving the mouth for respiration are $\mathrm{CO}_{2}, \mathrm{O}_{2}$ and $\mathrm{N}_{2}$ particles with actual concentrations (The air exiting from the lungs consists of $80 \%$ nitrogen, $16 \%$ oxygen and $4 \%$ carbon dioxide.) and also the particles that play the role of bacteria exit the respiratory tracts 
with an amount of 1000 particles per respiration. The temperatures of the input air and the output air from the respiratory tracts are considered respectively equal to the room initial temperature and the temperature of the human body under normal conditions.

\subsection{Mesh Independency and numerical validation}

Three types of meshes with 781836, 1109468 and 1674702 cells were generated in order to examine the mesh independency of the solution. The mesh is unstructured and non-conformal and it is to investigate the bacteria concentration at a distance of $5 \mathrm{~cm}$ from the mouth. The mesh with 1109468 cells will be used for the subsequent solutions because of providing mesh-independent solutions. The numerical results of the normalized concentration distribution in the Lagrangian method for the present study have been validated comparing with the experimental results of Chen et al. [9]. The details of this validation are not discussed here.

\section{The Results and Discussion}

One of the objectives of this project is to find out the number of respirations required for attaining the steady state condition. In the next stage, the mesh with 1109468 cells was selected according to the mesh independence study and employed in the subsequent simulation.

Five six-second respiratory cycles (30 seconds in the aggregate) were simulated in this stage. Figure 1 shows the periodic mass fraction in breathing cycles. According to the diagram shown in this figure, it can be observed that the bacteria concentration at a distance of $5 \mathrm{~cm}$ from the mouth has become periodic in the last two cycles after three respiration cycles $(2.5 \mathrm{sec}$. exhale, $2.5 \mathrm{sec}$. inhale and $1 \mathrm{sec}$. pause). The reason for disparity during the first 3 cycles was that the flow inside the room had not achieved the steady-state yet (started from stagnation state) and reaches the steady state after about 18 seconds. It is evident that the existence of a peak in each cycle pertains to the maximum output amount of bacteria at the end of the inhale stage.
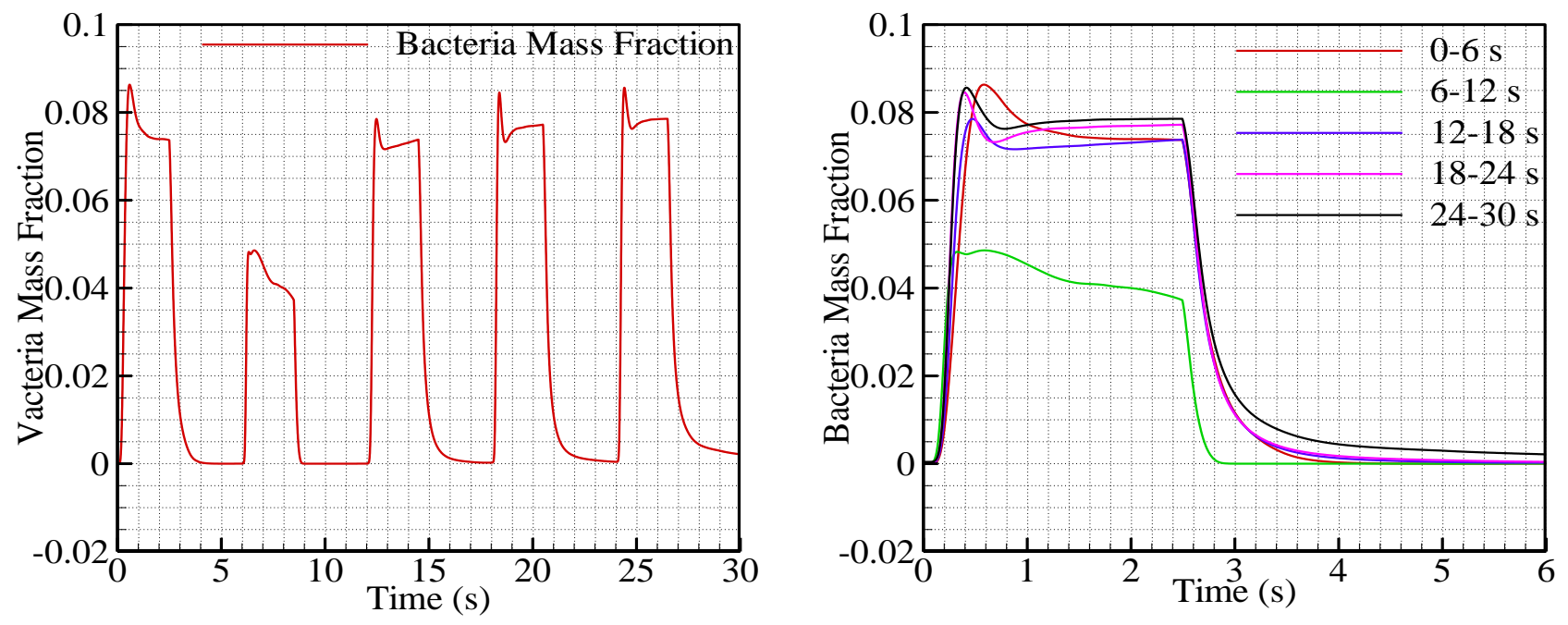

Fig. 1: Periodic mass fraction in breathing cycles.

Figure 2 shows the bacteria mass fraction concentration at mid-plane of cleanroom at three different times of 27.000, 28.875, and 29.375 seconds. According to the contours shown in this figure, when the steady state condition has been almost reached, we can see the concentration of bacteria accumulated around the patient's head during inhalation. The 
concentration value decreases over time, and its accumulation region will also move depending on the room input air velocity.

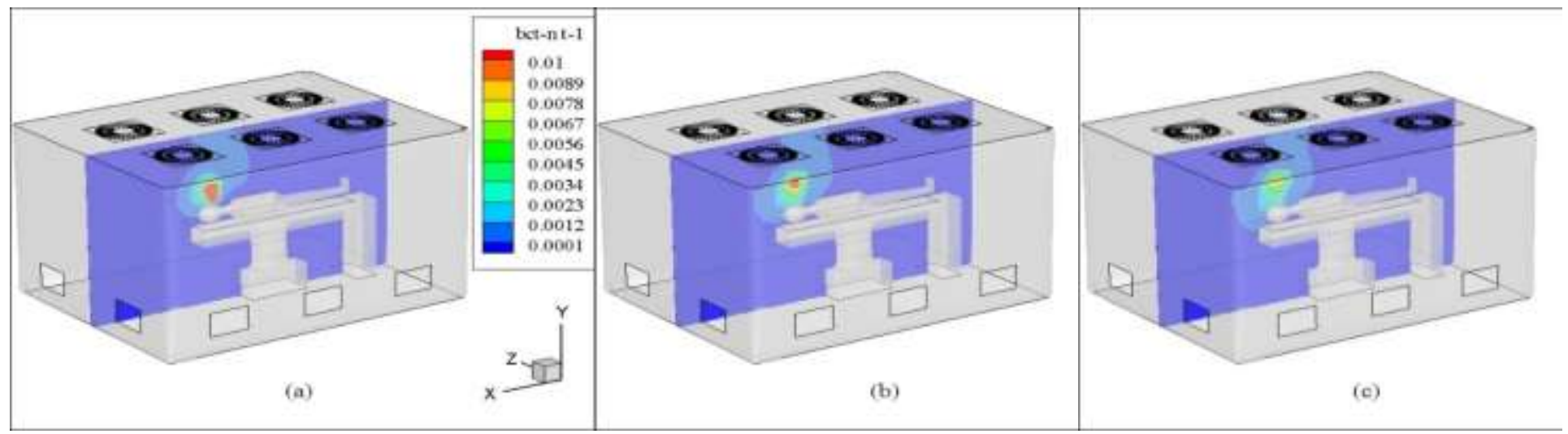

Fig. 2: Bacteria mass fraction concentration at mid-plane (a) $27 \mathrm{~s}$, (b) $28.875 \mathrm{~s}$ and (c) $29.375 \mathrm{~s}$.

\section{Conclusion}

In many hospital cleanrooms such as the open-heart surgery, organ transplantation and neurosurgery rooms, it is very important to disinfect the pollutant particles, which can lead to capillary clogging during an ordinary operation the bacteria concentration in the specified region has gradually decreased with time relative to the beginning moments. This is an important observation in the application of isolation rooms with special conditions to reduce the risk of transmitting airborne micro-organism to other vulnerable patients and hospital staff and due to the need to better control such contaminations. The current study showed that the concentration value would decrease with time, and its accumulation region would also move away depending on the room input air velocity.

\section{Acknowledgements}

The authors, hereby, express their gratitude for the financial support received from the Deputy of Research and Technology in Sharif University of Technology.

\section{References}

[1] M. Shimada, K. Okuyama, S. Okazaki, M. Matsukura, and Y. Ishizu, "Numerical simulation and experiment on the transport of fine particles in a ventilated room," Aerosol Science and Technology, vol. 25, no. 3, pp. 242-255, 1996.

[2] M. Saidi, B. Sajadi, and G. Molaeimanesh, "The effect of source motion on contaminant distribution in the cleanrooms," Energy and Buildings, vol. 43, no. 4, pp. 966-970, 2011.

[3] C. Méndez, J. San José, J. Villafruela, and F. Castro, "Optimization of a hospital room by means of CFD for more efficient ventilation," Energy and Buildings, vol. 40, no. 5, pp. 849-854, 2008.

[4] K. Zhong, X. Yang, Y. Kang, "Effects of ventilation strategies and source locations on indoor particle deposition," Building and Environment, vol. 45, no. 3, pp. 655-662, 2010.

[5] K. Hagström, A. Zhivov, K. Sirén, and L. Christianson, "Influence of the floor-based obstructions on contaminant removal efficiency and effectiveness," Building and environment, vol. 37, no. 1, pp. 55-66, 2002.

[6] H. Lee and H. Awbi, "Effect of internal partitioning on indoor air quality of rooms with mixing ventilation-basic study," Building and Environment, vol. 39, no. 2, pp. 127-141, 2004.

[7] X. Cao, J. Liu, N. Jiang, Q. Chen, "Particle image velocimetry measurement of indoor airflow field: A review of the technologies and applications," Energy and Buildings, vol. 69, pp. 367-380, 2014. 
[8] Z. Zhang, Q. Chen, "Comparison of the Eulerian and Lagrangian methods for predicting particle transport in enclosed spaces," Atmospheric Environment, vol. 41, pp. 5236-5248, 2007.

[9] F. Chen, S. C. M. Yu, A. C. K. Lai, "Modelling particle distribution and deposition in indoor environments with a new drift-flux model," Atmospheric Environment, vol. 40, pp. 357-367, 2006. 